Martin Kasparick*, Marcus Köny, Björn Andersen, Kathrin Riech, Anton Keller, Sven Kämmer, Andreas Guth, Lars Mündermann, Axel Stickel, Stephan Klöckner, Frank Golatowski, Dirk Timmermann

\title{
Service-Oriented Medical Device Connectivity: Particular Interoperability Standards for High Frequency Surgical Equipment and External Control Devices
}

\begin{abstract}
Manufacturer-independent medical device interoperability has been strongly demanded by the clinicians and healthcare delivery organizations (HDOs), however has not been achieved in practice for decades. The ISO/IEEE 11073 Service-oriented Device Connectivity (SDC) family of standards constitutes a paradigm shift. This work supplements SDC with Device Specializations (DevSpecs) or Modular Specifications (ModSpecs): particular interoperability standards for high frequency (HF) surgical equipment and external control devices, such as foot or finger switches. They provide models to describe these devices in a service-oriented medical device system and modes of interaction with other network participants. Additionally, we contribute to the ISO/IEEE 11073-10101 nomenclature standard to provide semantic descriptions of the exchanged information. This is a key enabler for safe and effective medical device interoperability to support the caregivers and improve patient safety as well as clinical outcome.
\end{abstract}

Keywords: high frequency (HF) surgical equipment, external control device, IEEE 11073 SDC, medical device interoperability, device specialization (DevSpec)

\footnotetext{
*Corresponding author: Martin Kasparick: Institute of Applied Microelectronics and Computer Engineering, University of Rostock, Germany, martin.kasparick@uni-rostock.de Marcus Köny: steute Technologies $\mathrm{GmbH}$ \& Co. KG, Löhne, Germany; Björn Andersen: Drägerwerk AG \& Co. KGaA, Lübeck, Germany; Kathrin Riech: Institute of Medical Informatics, University of Lübeck, Lübeck, Germany; Anton Keller: Aesculap AG, Tuttlingen, Germany; Sven Kämmer: BOWA-electronic GmbH \& Co. KG, Gomaringen, Germany; Andreas Guth: Erbe Elektromedizin $\mathrm{GmbH}$, Tübingen, Germany; Lars Mündermann, Axel Stickel: Karl Storz SE \& Co. KG, Tuttlingen, Germany; Stephan Klöckner: Olympus Winter \& Ibe GmbH, Hamburg, Germany; Frank Golatowski, Dirk Timmermann: Institute of Applied Microelectronics and CE, University of Rostock, Germany
}

https://doi.org/10.1515/cdbme-2021-2133

\section{Introduction}

The ISO/IEEE 11073 Service-oriented Device Connectivity (SDC) family of standards has been introduced to enable manufacturer-independent medical device interoperability. The focus is on the operating room (OR), intensive care unit (ICU), and professional clinical devices. The SDC Core Standards have been published and recognized. They comprise the communication technology (Medical Devices Communication Profile for Web Services, MDPWS), the domain information and service model (also called Basic Integrated Clinical Environment Protocol Specification, $B I C E P S)$, and the architecture and protocol for serviceoriented point-of-care medical device communication [1].

To ensure safe and effective interconnection and increase interoperability, the Participant Key Purpose (PKP) standards are currently being developed. The $P K P s$ specify requirements based on the roles of a participant. There are general requirements for all $S D C$ participants and more specific ones for those providing and/or consuming data, alerts, and external control capabilities [2].

SDC Core Standards and PKPs provide the technology for interoperable medical devices agnostic of the device type. Thus, plenty of freedom remains to model the $S D C$ representation of a particular device [3]. Consequently, different manufacturers tend to model functionally similar devices in greatly differing structures. Whereas this does not hinder safe data exchange, it heavily increases implementation complexity for service consumers [4]. Additionally, this flexibility increases the required approval effort.

The SDC Device Specialization (DevSpec) and Modular Specification (ModSpec) standards address these challenges. 
DevSpecs describe the network representation of particular medical devices, such as high frequency (HF) surgical equipment (see section 3). In contrast, ModSpecs are libraries of submodules representing functionally similar subsystems that are present in different device types, such as foot switches (see section 4).

The development of these standards started in the research project Modular Specialisations for Point-of-Care Medical Devices (PoCSpec) that brought device experts from the manufacturers and interoperability experts together to build consensus standards. PoCSpec focused on endoscopic devices [4] and high frequency (HF) surgical equipment.

This paper presents the current progress on the standard drafts IEEE P11073-10721 for HF surgical equipment and parts of IEEE P11073-10720 for external control devices. Additionally, there are extensive contributions to the ISO/IEEE 11073-10101 nomenclature.

We aim for the standards to be as strict as necessary to ensure safe and effective interoperability and as flexible as possible to allow for manufacturer-specific innovations. Thus, major parts of the models are optional, but if a device provides a particular optional functionality, it has to adhere to the model. The standards encourage to include as many features as possible into every device's network representation.

\section{IEEE 11073 SDC Modelling}

The DevSpecs and ModSpecs are specializations of the domain information and service model defined in ISO/IEEE 1107310207 (BICEPS). The device capabilities are modelled in a structure called containment tree. The root node of this tree with a maximum depth of four is the medical device system $(M D S)$ that represents the whole device. Physical or logical subsystems are described as virtual medical devices (VMDs). Channels are the third level that represent logical or physiological groupings of metrics that are the leaf nodes. Metrics model parameters, measurements, calculations, settings, etc. Additionally, alerts, external control capabilities, and contextual information are included in the network representation. For the sake of clarity, we omit many of these features in this paper and only address the most important metrics.

\section{HF Surgical Equipment}

High frequency (HF) surgical equipment (also known as radio frequency or electrosurgical equipment) is commonly used in many of the today's surgical interventions. HF surgical equipment delivers high frequency currents to perform surgical modification of tissue that is based on thermal effects. The most common forms of tissue modification are cutting and coagulation, but may also include tissue ablation, lesioning, shrinkage, sealing, or fusion.

Fundamentally, there are two HF application methods: monopolar and bipolar. Monopolar means applications with a small area active electrode of the instrument and a large area neutral electrode that is typically stuck to the patient's body. The intended thermal effect at the target tissue is induced as the current flow over the active electrode (small area) leads to a high current density. In contrast, the large area neutral electrode leads to a low current density and thus prevents the adjacent tissue from thermal effects. Typical monopolar instruments have the shape of a scalpel or a sphere. Bipolar applications use two active electrodes of the instrument where the intended thermal tissue effect takes place. There is no need for a separate neutral electrode. Typical examples are instruments like scissors or forceps.

The operator activates today's HF surgical equipment, i.e. initiates the delivery of HF current, by foot switches or finger switches on the handpieces of the instruments. The IEC 60601-2-2 enforces clear distinction between controls to activate the different HF surgical modes, e.g. yellow colour for cutting and blue colour for coagulation. Additionally, the activation can be triggered by tissue contact that is detected by the devices. Releasing the foot or finger switch stops the activation. In some configurations, the HF current delivery stops automatically based on tissue effect sensing or timing.

\subsection{Operational Concepts}

The operational concepts of today's HF surgical equipment can be divided into active output terminal-oriented and active accessory-oriented.

The active output terminal is the connection jack or plug socket of the HF generator where the cable of the instrument (active accessory) is plugged in. Active output terminaloriented means that the operational parameters are directly related to the active output terminal. As the configuration is typically done for a particular instrument, it has to be ensured that the intended instrument is connected to the configured connection jack. In contrast, active accessory-oriented means that the user directly configures the operational parameters of the instrument. This is independent from where and even whether the active accessory is plugged into an active output terminal. The HF generator recognizes the pairing of active accessory and active output terminal and behaves accordingly. 


\subsection{Concept of Virtual Terminals}

A model that represents HF surgical equipment in an $S D C$ network has to cover both operational concepts. It is our goal to have a uniform model that is independent from whether the active accessory or the active output terminal is the leading concept for the user's interaction. Therefore, we introduce an abstraction: the Virtual Terminal.

The term Virtual Terminal is derived from the active output terminal. However, as the physical assignment between active output terminal and active accessory is not necessarily present, we call this abstraction virtual.

The Virtual Terminal is a basic building block of our device model. According to the BICEPS domain information model it is represented as a virtual medical device (VMD), illustrated in the blue part of Figure 1). As the Virtual Terminal contains information about active accessory and active output terminal, it covers aspects of monopolar and bipolar $\mathrm{HF}$ application method and of HF surgical modes cutting and coagulation. Thus, the four resulting combinations are modelled as channels in the containment tree. They contain several parameters, e.g. output settings (maximum delivered output power, type of HF surgical mode, effect), optional parameters to configure automatic activation/deactivation, measurements and calculations such as the actually delivered output power, etc.

Additionally, there is a channel that groups information about the instrument and an optional channel for argon-based application parameters.

\subsection{Auxiliary Containment Tree Entries}

The VMD neutral electrode is mandatory for all devices providing a monopolar HF application method. Depending on the capabilities, it contains information about the neutral electrode itself, the continuity monitoring, and the contact quality monitoring (see also Figure 1). This also includes alerting functionalities.
General device settings are covered by a particular VMD. This can include user interface settings, like display brightness, language, sound volume, etc. Additionally, globally applicable settings are modelled, for example, the maximum activation time if it is not configurable per Virtual Terminal.

The following parts will be based on ModSpecs that are referenced. Surgical devices commonly provide different programs for different surgical specialties or procedures. The chosen program influences device parameters, which can be modelled using the BICEPS relation mechanisms.

If the HF surgical equipment provides argon-based applications, the supply with argon gas is modelled using the generic gas supply ModSpec.

If multiple instruments are attached/configured, the user can manage the association between the foot pedals and the intended functionality activation of the intended instrument (within the boundaries of IEC 60601-2-2). The given configuration and potentially re-configuration capabilities will be realized according the ModSpec external control devices (see section 4). A hardwired foot switch is currently only capable of associations to functionalities of the device it is connected to. Thus, first implementations will only utilize the internal association feature of the ModSpec.

\section{External Control Devices}

In this paper, we focus on the $S D C$ representation of external control devices, e.g. foot switches, and external control items, e.g. pedals of foot switches, that fulfil the service provider role. The actual external control potentially realized by the consumer part is out of scope of this paper.

According to the principles described in [5], [6], there are two main aspects: the item state (e.g. for a simple foot pedal whether it is currently pressed or released) and the association state that describes which functionality of which device shall be triggered when the control element is used.

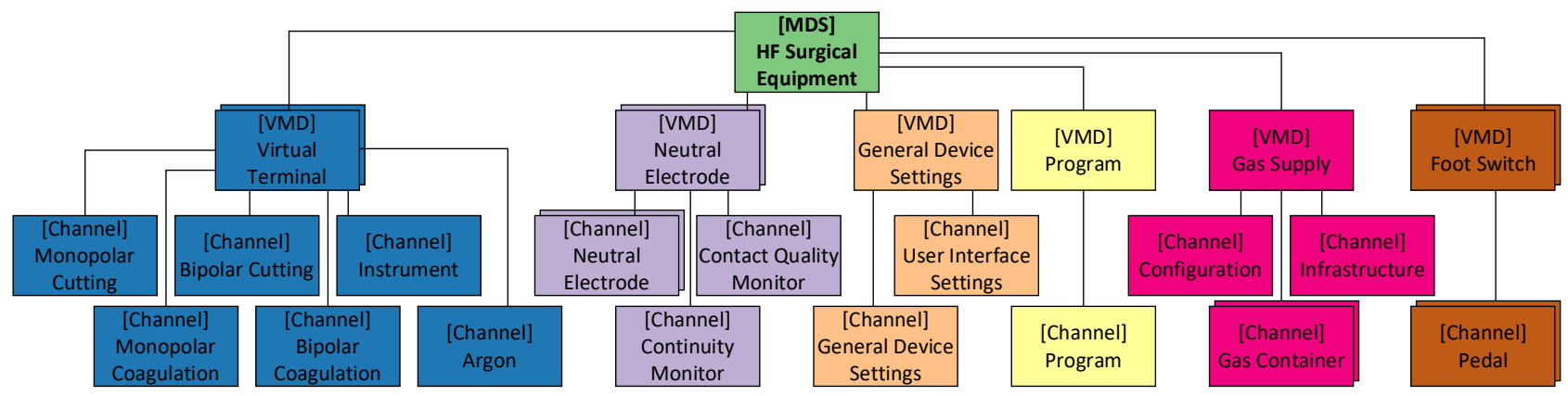

Figure 1: Containment tree structure of HF surgical equipment (without metrics); stacked boxes indicate potentially multiple instances. 
External control devices are commonly used by different types of medical devices. Thus, we standardize these aspects as a ModSpec that is part of the standard proposal IEEE P11073-10720.

\subsection{Modelling Item State}

The item state is modelled by at least one metric depending on the dimension of the state and the type. For example, a discrete foot switch pedal is modelled by an enumeration metric which represents the discrete values of the state, e.g. released or pressed. A more complex item like a joystick can be modelled by two numeric metrics for the direction and the amplitude.

\subsection{Modelling Associations}

We distinguish between internal and external association: Internal association means that it is limited to functionalities of the device the external control device is directly connected to (without using the SDC network). External association means that functionalities can be associated that are provided by other participants in the $S D C$ network by offering external control operations. An internal association can be clearly defined by referencing an operation within the device description. In case of external associations, a unique identifier of the corresponding participant is added by using its endpoint reference. The tuple describing the association is furthermore completed with state version information.

The tuple of metrics is grouped in a channel per assignable state value. For example, if a foot pedal has three state values, released, half-pressed, and full-pressed, it is possible to describe up to three associations, although typically the released state association is not used as no operation is triggered. Taking complex interaction devices such as joysticks into account demonstrates the complexity of the representation. Thus, standardized models ease the burden of implementation and approval for providers and consumers.

\section{Conclusion}

We presented the current state of the Device Specialization (DevSpec) and Modular Specification (ModSpec) draft standards for high frequency surgical equipment and external control devices (IEEE P11073-10721 and parts of -10720) that supplement the ISO/IEEE 11073 SDC standards family. This work will further increase interoperability and reduce implementation and approval efforts for these devices and interconnected $S D C$ participants. The presented models cover the common consensus features and operational concepts. Future extension and manufacturer-specific innovations are possible and foreseen. Thus, this work supports the vision of fully manufacturer-independently integrated medical device systems and assistive systems based thereon.

The current state of work can be viewed and discussed publicly [7]. The standard drafts are being discussed in international working groups and improved based on the feedback. The standard ballot will start upon successful ballot of the SDC PKP standards. Additional DevSpecs and ModSpecs are planned beyond the PoCSpec project, e.g. for medical devices in the anaesthesia domain.

\section{Author Statement}

Research funding: This work was partially supported by the German Federal Ministry for Economic Affairs and Energy (grant number: 03TNH017).

\section{References}

[1] M. Kasparick, M. Schmitz, B. Andersen, M. Rockstroh, S. Franke, S. Schlichting, F. Golatowski, and D. Timmermann, "OR.NET: a service-oriented architecture for safe and dynamic medical device interoperability," Biomed. Eng. / Biomed. Tech., vol. 63, no. 1, pp. 11-30, Feb. 2018, doi: 10.1515/bmt-2017-0020

[2] M. Kasparick, B. Butzin, F. Golatowski, J. Pabst, H.-J. Cappius, P. Westerhoff, B. Andersen, and D. Timmermann, "From IEEE 11073 SDC Device Specializations to Assistive Systems: Rule-based Data Analysis for Minimal Invasive Surgery," IEEE SmartNets, 2019, pp. 1-7, doi: 10.1109/SmartNets48225.2019.9069774

[3] B. Andersen, S. Baumhof, and J. Ingenerf, "Service-Oriented Device Connectivity: Device Specialisations for Interoperability," Studies in health technology and informatics: MEDINFO, 2019, pp. 509-511, doi: 10.3233/SHTI190274

[4] B. Andersen, M. Kasparick, K. Riech, S. Klöckner, A. Keller, L. Mündermann, J. Maier-Holzberg, D. Timmermann, and J. Ingenerf, "Service-Oriented Medical Device Connectivity: Particular Standards for Endoscopic Surgery," IEEE EMBC, 2020, doi: 10.1109/EMBC44109.2020.9175932

[5] M. Kasparick, M. Schmitz, F. Golatowski, and D. Timmermann, "Dynamic remote control through service orchestration of point-of-care and surgical devices based on IEEE 11073 SDC," IEEE HI-POCT, 2016, pp. 121-125, doi: $\underline{10.1109 / H I C .2016 .7797712}$

[6] M. Kasparick, "Zuverlässige und herstellerübergreifende Medizingeräteinteroperabilität - Beiträge zur IEEE 11073 SDC-Normenfamilie," Dissertation University of Rostock, 2021, doi: $10.18453 /$ rosdok id00003032

[7] PoCSpec Project, "SourceForge Plattform: Modular Specialisations for Point-of-Care Medical Devices," https://sourceforge.net/projects/pocspec (accessed 12.07.2021) 